Publicación Semestral Pädi No. 10 (2018) 87-90

\title{
Situaciones Didácticas en Educación Matemática
}

\author{
Fernando Barrera-Mora a , Aarón Reyes-Rodríguez a, \\ a Área Académica de Matemáticas y Física, Universidad Autónoma del Estado de Hidalgo, 42184 Mineral de la Reforma, Hidalgo, México.
}

\section{Resumen}

Describimos los aspectos principales de la teoría de situaciones didácticas en educación matemática. En particular, caracterizamos esta aproximación a la didáctica en términos del tipo de tareas que propone, las características del proceso de instrucción y la función del profesor. Este enfoque no tiene un carácter prescriptivo, sino más bien plantea opciones interesantes que pueden modificarse y adaptarse en función de las necesidades de cada salón de clase.

Palabras Clave: aproximación didáctica, entendimiento matemático, tareas, proceso de instrucción, actividad del profesor.

\section{Introducción}

De acuerdo con el diccionario de la lengua española (RAE, 2014) y el diccionario de uso del español (Moliner, 2001), la didáctica es el arte de enseñar, es aquello relativo o perteneciente a la enseñanza. También se ha conceptualizado como un conjunto de técnicas propias de la enseñanza (Abbagnano y Visalberghi, 1992). En este trabajo consideramos que la didáctica es la disciplina cuyo objeto de estudio son los procesos de enseñanza-aprendizaje y sus actores, consecuentemente la actividad central consiste en reflexionar acerca de los principios, métodos, componentes y variables involucrados en la práctica educativa. Asimismo, concebimos a la didáctica de las matemáticas como la disciplina que estudia las condiciones para la diseminación y apropiación del conocimiento matemático en las instituciones educativas (Artigue et al., 2014).

De esta concepción surgen preguntas tales como: ¿para qué se enseña? ¿Qué enseñar? ¿Cómo enseñar un tópico? ¿Qué elementos influyen en la enseñanza-aprendizaje? ¿Cómo organizar el escenario de instrucción y sus implicaciones? ¿Cómo lograr los objetivos de instrucción?

Cada aproximación didáctica responde a estas preguntas de una forma particular. En este trabajo realizamos una revisión de las características más relevantes de la aproximación denominada Teoría de Situaciones Didácticas (TSD), enfatizando aspectos correspondientes a tres variables: el tipo de tareas, los procesos de instrucción y el rol del profesor. Los elementos descritos no tienen un carácter prescriptivo, es decir, no indican lo que el profesor debe hacer, sino más bien son opciones que, con base en un análisis de las características de los estudiantes y de algún grupo específico, pueden modificarse y adaptarse en función de ciertas necesidades.

\section{Teoría de Situaciones Didácticas}

Esta aproximación fue desarrollada por Guy Brousseau en los años setenta del siglo pasado. Constituye una aproximación sistémica al proceso de instrucción que se lleva a cabo en un salón de clase, incluyendo las interacciones en el aula, el proceso de planificación y la forma de mejorar este proceso (Simon, 2013). En esta propuesta, las tareas se denominan situaciones a-didácticas. Éstas se caracterizan por plantearse en un contexto lúdico y no hacer explícita una intención de enseñanza, es decir, el profesor no menciona a los estudiantes cuáles son los contenidos que se abordarán. Se espera que la situación a-didáctica promueva las acciones, reflexiones y discusiones entre los estudiantes sin la intervención del profesor (Warfield, 2014) y que los conceptos matemáticos emerjan a través de los procesos y acciones que los estudiantes desarrollan; permitiendo la construcción de significados estructurados para esos conceptos. En una situación adidáctica, el contexto de juego debe permitir que el conocimiento aparezca como solución o como el medio para establecer la estrategia óptima. Un ejemplo paradigmático de situación a-didáctica es el juego de la carrera a 20 (Brousseau, 1997).

En la "carrera a 20" compiten dos estudiantes. En una tabla con dos columnas se anotan los resultados del juego. Por medio de un volado, u otro mecanismo similar, se decide cuál de los dos jugadores inicia la partida. El primer participante debe elegir un número, que puede ser uno o dos. Una vez realizada su elección, dice el número en voz alta y lo anota en la tabla. El otro participante debe sumar uno o dos al número del primer participante, a continuación dice el resultado de la suma en voz alta y lo anota en la tabla. El objetivo del juego es continuar este procedimiento hasta que uno de los dos jugadores obtenga como resultado 20 . El estudiante que llega a 20 es el ganador de la ronda. Por ejemplo, supongamos que el primer jugador dijo uno, entonces el segundo jugador sumó

* Autor en correspondencia. 
dos a ese número, obteniendo tres como resultado. A continuación, el primer jugador sumó dos al resultado previo y obtuvo cinco como nuevo resultado, y así sucesivamente (Tabla 1).

Tabla 1: Registro de algunos resultados de una partida de la carrera a 20.

\begin{tabular}{cc}
\hline Jugador 1 & Jugador 2 \\
\hline 1 & 3 \\
5 &
\end{tabular}

El proceso de instrucción se organiza en cuatro fases (o Situaciones como las llama Brousseau): (i) fase de acción, (ii) fase de formulación, (iii) fase de validación y (iv) fase de institucionalización. En primer lugar, el profesor explica cuáles son las reglas del juego y ejemplifica cómo jugar. Posteriormente, pide a los estudiantes que jueguen por parejas (fase de acción), mientras que él sólo interviene para resolver dudas respecto de las reglas del juego y para observar que se mantenga el orden en el salón de clase. Al llevarse a cabo diferentes rondas, algunos estudiantes advertirán, tal vez inconscientemente, que si llegan a 17, entonces ganan (se recomienda permitir la realización de cuatro o cinco rondas).

Después de concluir el juego por parejas, el profesor organiza a los estudiantes en dos equipos. Se elige, de forma aleatoria, un representante para cada equipo en cada una de las rondas (se recomienda realizar seis u ocho). Los representantes juegan, anotando los resultados de la ronda en el pizarrón (nueva fase de acción). El ganador de cada ronda suma un punto para su equipo. Los estudiantes se darán cuenta que es necesario que compartan y discutan entre ellos las posibles estrategias que les permitan ganar (fase de formulación), por ejemplo, pueden sugerir que los representantes deben tratar de llegar al 17 o al 14 para ganar.

Finalizada la competencia por equipos, el profesor solicita a los estudiantes que registren, por escrito, sus conjeturas respecto de las estrategias que les permitieron ganar (nueva fase de formulación) y pide a cada equipo, de forma alternada, que mencione en voz alta uno de sus descubrimientos. Entonces el profesor anotará la conjetura en el pizarrón. En esta fase es importante la construcción de un lenguaje que todos los integrantes del grupo puedan comprender. Cada vez que se comparte una conjetura (por ejemplo, el que llega a 17 gana), el equipo contrario debe aceptarla o rechazarla como válida. Si la conjetura se acepta, cambia su estatus por el de teorema, permaneciendo en el pizarrón y, en caso contrario, se borra. Para apoyar la aceptación de una conjetura (fase de validación) el estudiante que la propuso debe ofrecer argumentos ("dije varias veces 17 y gané" o "si digo 17, mi oponente puede decir 18 o 19, y entonces yo digo 20 y gano") de por qué la conjetura es cierta, pero el equipo contrario también puede contra argumentar ("yo dije una vez 17 y perdí"). Los estudiantes aportan argumentos hasta que se logra un consenso para aceptar o rechazar la conjetura. Cada conjetura aceptada como verdadera brinda un punto al equipo que la propuso, mientras que cada conjetura refutada permite ganar tres puntos al equipo que la refutó.

Se espera que los estudiantes lleguen a conjeturar que la estrategia ganadora consiste en elegir el número 2, y posteriormente sumar consecutivamente, entre él y su oponente, 3 a este número para obtener la secuencia $5,8,11$, $14,17,20$, lo cual siempre es posible independientemente de las acciones que tome el segundo jugador. Entonces, el ganador será el jugador que inicia el juego, siempre y cuando siga la estrategia ganadora. Al finalizar este primer juego aún no será claro cuál es el contenido objetivo, para que emerja con mayor claridad el concepto matemático es necesario pedir a los estudiantes que jueguen variaciones de la carrera 20 , por ejemplo carrera 28 , carrera 36 . En la carrera 28 , los estudiantes podrán darse cuenta que gana el jugador que diga el número 1 y después siga la secuencia de números $4,7,10,13,16,19$, $22,25,28$. En la carrera 36 gana el jugador que dice el número 3 y sigue la secuencia $6,9,12,15,18,21,24,27,30,33,36$. En este último caso gana el segundo jugador, siempre que siga la estrategia ganadora.

Además, es necesario variar los números que cada jugador puede avanzar, por ejemplo se puede jugar carrera 20, pero ahora permitir que los avances sean 1, 2 o 3 . En este caso gana el jugador que dice el número 4 y sigue la secuencia $8,12,16$, 20. Por lo tanto, conociendo esta estrategia, el segundo jugador siempre gana el juego.

La finalidad de jugar esta diversidad de carreras es que los estudiantes descubran cómo obtener la estrategia ganadora para la "carrera $n$ " con avances $1,2, \ldots, k(\operatorname{con} k<n)$. Los estudiantes podrán conjeturar que el residuo de la división de $n$ entre $k+1$ indica el número que se debe elegir de inicio para ganar, siempre que este residuo sea diferente de cero, mientras que el divisor es el número que se debe sumar al número inicial para obtener la secuencia ganadora. En caso de que el residuo de la división sea cero, gana quien elija primero el número $k+1$. Desde luego, no se espera que estudiantes de primaria expresen simbólicamente la conjetura general, pero sí que puedan expresarla verbalmente. Sin embargo, los profesores están obligados a resolver, de forma previa, la tarea en su nivel de mayor generalidad, antes de intentar implementarla con estudiantes.

En la fase de institucionalización, el profesor debe aclarar cuál fue la finalidad de las tareas que se desarrollaron, así como precisar los términos o conceptos matemáticos que emergieron, ayudar a los estudiantes a distinguir las ideas correctas y las falsas, y situar a los conceptos en el contexto del currículo escolar. En el caso de la carrera 20, se debe aclarar que el concepto básico es el de progresión aritmética y su vez, ésta incluye al algoritmo de la división de números enteros, así como terminología relacionada: divisor, dividendo, residuo y cociente. Como menciona Brousseau (1997, p. 3), el objetivo de la carrera 20 es "revisar la división en circunstancias en las cuales el 'significado' de la operación no coincide con el aprendido previamente y fomentar el descubrimiento y demostración, por parte del estudiante, de una sucesión de teoremas".

Es importante que el profesor registre las principales ideas de los estudiantes para proporcionarles retroalimentación de aquello que han generado o descubierto y cómo esto se relaciona con sus conocimientos previos, con la finalidad de construir los puentes entre los conocimientos desarrollados por los estudiantes y los conceptos institucionalizados. Durante la fase de institucionalización el profesor puede introducir una notación convencional o introducir terminología estándar, en caso de que lo considere pertinente.

Una condición para que el estudiante aprenda, desde la perspectiva de situaciones didácticas, es que acepte la tarea como suya y actúe en consecuencia (haciendo, pensando, reflexionando o comunicando ideas). El conocimiento es resultado de la adaptación del estudiante a un milieu el cual genera contradicciones, dificultades y desequilibrios, siendo las respuestas que el estudiante proporciona, evidencia del 
aprendizaje (Brousseau, 1997, p. 30). El término milieu hace referencia a todo aquello que influye sobre el estudiante y aquello sobre lo que el estudiante influye en cada fase de implementación de la tarea. No traducimos milieu como medio, ya que existen diferencias de interpretación que se aclararán con un ejemplo.

En la fase de acción del juego por parejas, el milieu de cada jugador está integrado por su contrincante y los datos de la partida, registrados en la tabla, porque a partir de la información que le ofrecen las acciones del contrincante y los datos de la tabla, el estudiante toma decisiones sobre cómo actuar. En este caso ni el profesor ni el resto de los estudiantes son parte del milieu, aunque sí del medio que constituye el salón de clase. Durante la fase de formulación, llevada a cabo cuando cada equipo discute las estrategias que debe implementar su representante, el milieu de cada estudiante está integrado por sus compañeros de equipo, todas las rondas que se han jugado y particularmente, los resultados de la última ronda anotados en el pizarrón.

La función del profesor, desde la perspectiva de las situaciones didácticas, consiste en seleccionar, diseñar o rediseñar una situación a-didáctica que encapsule cierto concepto matemático, así como organizar un ambiente para simular una micro-sociedad científica, alentar a los estudiantes a que formulen preguntas, solucionen controversias entre ellos, apoyar la construcción de un lenguaje apropiado y orientar los criterios de justificación apropiados para un aula en particular. En esta aproximación no es tarea del profesor mostrar a los estudiantes como resolver el problema o desafío que supone abordar la tarea (Radford, 2008).

Una desventaja de esta aproximación es que la mayoría de las situaciones a-didácticas que se han propuesto se enfocan en conceptos que se revisan en la educación básica, particularmente entre cuarto grado de primaria y segundo de secundaria (Warfield, 2014). Por otra parte, se ha reconocido la dificultad para encontrar situaciones a-didácticas apropiadas para el aprendizaje de diversos conceptos sobre todo los que se abordan en el nivel superior (Grønbæk et al., 2009). Esta dificultad se debe, principalmente, al nivel de abstracción de los conceptos que se revisan en el nivel universitario, así como al nivel de organización y las interrelaciones entre estos, además de la cantidad de conocimientos previos necesarios para abordarlos y entenderlos (González-Martín, et al., 2014).

A pesar de lo anterior, existen ejemplos paradigmáticos de la utilización, si no de la teoría en su totalidad, sí de algunos conceptos tales como milieu, contrato didáctico o las fases de acción, formulación, validación e institucionalización; para promover el aprendizaje de temas entre los que destacan ecuaciones diferenciales (Artigue, 1994) o integrales (Legrand, 2001).

Otra dificultad para implementar a las situaciones didácticas en el ámbito escolar, radica en que los profesores no entiendan las ideas centrales de la propuesta, así como la teoría que las sustenta, y centren la atención únicamente en el juego, como ocurrió en México a finales de la década de los 80 y principios de los 90 del siglo pasado, cuando se incluyó la carrera 20 en algunos materiales educativos (ver como ejemplo Fuenlabrada et al., 1994).

El profesor puede experimentar dificultades al tratar de implementar la tarea, porque el contrato didáctico (es decir, expectativas mutuas, no necesariamente explícitas, entre el profesor y los estudiantes respecto de su papel en los procesos de enseñanza-aprendizaje) imperante en su salón de clase no es el apropiado, por ejemplo, cuando el estudiante considera que es obligación del profesor explicar cómo resolver los problemas y que el papel de los estudiante es resolver problemas parecidos a los que explicó el maestro. En estos casos, los estudiantes pueden externar opiniones del tipo "yo vengo a que usted me enseñe cómo resolver los problemas, no a que yo le enseñe a usted cómo resolverlos". El obtener buenos resultados con esta aproximación requiere de un contrato didáctico donde los estudiantes aceptan la responsabilidad de resolver problemas cuya solución no se les ha enseñado previamente, y aceptar que siempre es posible obtener una solución aunque no puedan identificar la ruta que deben seguir desde el principio (Warfield, 2014).

\section{Reflexiones Finales}

Conocer diferentes aproximaciones didácticas es un aspecto importante en la formación de todo profesor de matemáticas, ya que este conocimiento le permitirá contar con diversos elementos para abordar los múltiples retos presentes en la práctica educativa. Durante su actividad docente, el profesor de matemáticas es, o debiera ser, un bricoleur, es decir una persona práctica e ingeniosa, quien resuelve las dificultades que se le presentan al utilizar y adaptar los materiales que tiene a la mano, e incluso al inventar nuevas aplicaciones para los materiales o herramientas que utiliza (Gravemeijer, 1998). Específicamente, los profesores de matemáticas enfrentan el reto de adaptar sus conocimientos didácticos a las necesidades particulares de cada uno de los salones de clase en los que trabajan, más que adherirse a una sola perspectiva, la cual puede ser insuficiente para dar cuenta de la complejidad de los procesos de enseñanza-aprendizaje.

Aquellos profesores interesados en conocer más sobre el tipo de tareas o secuencias que giran en torno de las situaciones didácticas, para promover el redescubrimiento y aprendizaje de ideas matemáticas tales como conteo, números racionales y probabilidad y estadística, pueden consultar las siguientes referencias: Brousseau (2000), Brousseau et al. (2014), Brousseau et al. (2002).

\section{English Summary}

\section{Didactical Situations in Mathematics Education.}

\section{Abstract}

We describe the main aspects of didactical situations in mathematics education. Particularly, we characterize this approach in terms of the type of tasks proposed there, the characteristics of the instructional process, and the teacher's activity. This paper does not have a prescriptive approach, it rather proposes interesting features that can be modified and adapted according to each classroom's needs.

\section{Keywords:}

Didactical approach, mathematical understanding, tasks, instructional process, teacher activity 


\section{Referencias}

Abbagnano, N., Visalberghi, A., 1992. Historia de la Pedagogía (novena reimpresión). Fondo de Cultura Económica, Madrid.

Artigue, M., 1994. Didactic engineering as a framework for the conception of teaching products. En: Biehler, R., Scholz, R.W., Sträßer, R., Winkelmann, B. (Eds.), Didactics of Mathematics as a Scientific Discipline. Kluwer Academic Publishers, Dordrecht, pp. 27-39.

Artigue, M., Aspekian, M., Corblin-Lenfant, A., 2014. Introduction to the Theory of Didactical Situations (TDS). En: Bikner-Ahsbahs, A., Prediger, S. (Eds.), Networking of Theories as a Research Practice in Mathematics Education: Advances in Mathematics Education, Springer, Cham, pp. 47-66.

Brousseau, G., 1997. Theory of Didactical Situations in Mathematics. Kluwer, Dordretch.

Brousseau, G., 2000. Educación y didáctica de las matemáticas. Educación Matemática 12(1), 5-38.

Brousseau, G., Brousseau, N., Warfield, V., 2002. An experiment in the teaching of statistics and probability. Journal of Mathematical Behavior 20 (3), 363-411.

Brousseau, G., Brousseau, N., Warfield, V., 2014. Teaching fractions through situations: A fundamental experiment. Springer, Dordrecht.

Fuenlabrada, I., Block, D., Balbuena, H., Carbajal, A., 1994. Juega y aprende matemáticas: Actividades para divertirse y trabajar en el aula (serie Libros del Rincón). SEP, México.

González-Martín, A. S., Bloch, I., Durand-Guerrier, V., Maschietto, M. 2014. Didactic Situations and Didactical Engineering in university mathematics: cases from the study of Calculus and proof. Research in Mathematics Education 16(2), 117-134
Gravemeijer, K., 1998. Developmental research as research method. En: Sierpinska, A., Kilpatrick, J. (Eds.), Mathematics Education as a Research Domain: A Search for Identity. Kluwer, Dordretch, pp. $277-$ 295.

Grønbæk, N., Misfeldt, M., Winsløw, C., 2009. Assessment and contract like relationships in undergraduate mathematics education. En: Skovsmose, O., Valero, P., Christensen, O. R. (Eds.), University Science and Mathematics Education in Transition. Springer, New York, pp. 85-105.

Legrand, M., 2001. Scientific debate in mathematics courses. En: Holton, D. (Ed.), The Teaching and Learning of Mathematics at University Level: An ICMI study. Kluwer Academic Publishers, New York, pp. 127-135.

Moliner, M., 2001. Diccionario de uso del español (versión electrónica 2.0). Gredos, Madrid.

Radford, L., 2008. Theories of mathematics education: A brief inquiry into their conceptual differences. Working paper, June 2008 prepared for the ICMI Survey team7: The notion and role of theory in mathematics education research. Recuperado el 21 de junio de 2017 de http://www.luisradford.ca/pub/31_radfordicmist7_EN.pdf

Real Academia Española [RAE], 2014. Diccionario de la lengua española $\left(23^{\mathrm{a}}\right.$ ed.). Espasa, Madrid.

Simon, M. A., 2013. The need for theories of conceptual learning and teaching of mathematics. En: Leatham, K. R. (Ed.), Vital directions for mathematics education research. Springer, New York, pp. 95-118.

Warfield, V., 2014. Invitation to didactique. Springer, Tokyo. 